\title{
Performance Prediction of Solar Collector Adsorber Tube Temperature using a Nonlinear Autoregressive Model with eXogenous Input
}

\author{
M. P. Islam \\ Biomechanical Systems \\ Ehime University, 3-5-7 Tarumi \\ Matsuyama 790-8566, Japan
}

\author{
T. Morimoto \\ Biomechanical Systems \\ Ehime University, 3-5-7 Tarumi \\ Matsuyama 790-8566, Japan
}

\begin{abstract}
This study examines modeling and simulation of the transient thermal behavior of a solar collector adsorber tube. The data used for model setup and validation were taken experimentally during the start-up procedure of a solar collector adsorber tube. ANN models are developed based on the nonlinear autoregressive with exogenous input NARX model and are implemented using the MATLAB ${ }^{\circledR}$ tools including the Neural Network Toolbox ${ }^{\mathrm{TM}}$. It is considered that the data used for model training and validation are experimental data taken during solar collector operation using standard instrumentation. The neural network predictions agreed well with experimental values with mean squared error which are near 0 and the best fit between outputs and targets (R) are very close to 1 . These results showed that NARX models (1-12-1 with $\mathrm{d} 1=10, \mathrm{~d} 2=9$ and 35 epochs) can successfully be used to predict thermal performance of the adsorber tube.
\end{abstract}

\section{General Terms}

Neural networks, training algorithm.

\section{Keywords}

Solar radiation, solar collector, adsorber tube temperature, neural network.

\section{INTRODUCTION}

Solar energy has various advantages over conventional energy sources, such as short installation time and long-life operation, simplicity, no moving parts, silent, safe, non-polluting, and it is renewable source of heat and electricity [1]. Solar-powered adsorption refrigerators in recent years have been receiving much attention as a replacement for conventional vapor compression refrigeration cycles driven by electricity. In an adsorption system, adsorbent (activated carbon) is used to adsorb methanol and promote its evaporation using a flat-plate solar collector. Solar collectors are a special kind of heat exchanger that transforms solar radiation energy to the internal energy of the transport medium [2]. These transforming processes depend on climatic factors such as incoming solar radiation, duration of sunlight, and the thermal properties of the collector adsorbing tubes, glazing materials, heat insulators, and transparent cover.

An Artificial Neural Network (ANN), inspired by the biological nervous system, unifies a series of learning and autocorrecting functions to resolve diverse problems using algorithms or computational programming codes. The processing elements of a neural network, first proposed by McCulloch and Pitts [3], are analogous to the neurons of a nervous system. An ANN-based approach is considered to be a very effective modeling method, because it can trace back during the training procedure. As a result, an ANN have ability tocan recognize highly complex, ill-defined time series patterns and nonlinear characteristics with better accuracy over other methods in making predictions $[4,5]$. Yang [6] stated that there are several significant reasons why ANNs are such a powerful tool for modelling dynamic systems:

(1) ANNs can accurately recognize the inherent relationship between any set of inputs and outputs without a physical model or even without information about internal behaviour, and yet ANN results account for all the physics relating the output to the input. This ability is essentially independent of the complexity of the underlying relationship such as nonlinearity, multiple variables, and parameters. This essential ability is known as pattern recognition as the result of the learning process.

(2) The methodology is inherently fault tolerant, due to the large number of processing units in the network undergoing massive parallel data processing.

(3) The learning ability of ANNs means the methodology can adapt to changes in parameters. This ability enables the ANN to also handle time-dependent dynamic modelling.

Lin et al. [7] showed that a NARX network behaves better with long-term dependencies by using a single neuron network compared with a recurrent network trained by Backpropagation through time (BPTT), and NARX networks have been tested for finite automata prediction. In theory, a NARX network can replace any recurrent network that is currently being used for a problem, without any computational power loss. A NARX model has also been shown to be effective for other types of chaotic series [8] (Diaconescu, 2008), including the chaotic Mackey-Glass series and Fractal Weierstrass series. For these series, a good prediction has been found with an average input order and low output order. The model has predicted values with $99 \%$ accuracy, comparing them with the original values. For a complex system, NARX has been shown again to have good results, with Bayesian regularization and in this scenario, the accuracy was $96 \%$.

In this study, solar radiation and collector adsorber tube temperature are considered the basic input-output parameter for nonlinear autoregressive models with an exogenous input (NARX) network to predict the collector temperature of a solar adsorption refrigerator. Therefore, this study included parameter identification (training), comparisons between observed and modelled collector output (estimated temperature), and simulated solar collector adsorber tube temperature. 


\section{MATERIALS AND METHODS 2.1 Construction Principle of the Solar Collector}

The construction of a flat-plate adsorption solar collector with a polystyrene foam casing, blackened absorber surface, and transparent cover sheets is shown in Fig. 1. The solar collector is mounted on an adjustable stand with an inclined angle of $32^{\circ} \mathrm{C}$ for winter, $56^{\circ} \mathrm{C}$ for spring/autumn, and $80^{\circ} \mathrm{C}$ for summer toward the horizon and oriented to the south. The dimensions of the rectangular casing are $1700 \times 1000 \times 300 \times$ $50 \mathrm{~mm}$ (length $\times$ width $\times$ height $\times$ thickness) with thermal conductivity $\lambda=0.034$ (W/m.K). The bottom and the side of the casing are well insulated by Rockwool ( $30 \mathrm{~mm}$ thick) with thermal conductivity $\lambda=0.040$ (W/m.K). The insulated materials provide sealing and reduce heat loss from the back or sides of the collector.

The collector plate is covered with a high transmittance Plexiglas plate, $1940 \times 940 \times 3 \mathrm{~mm}$ (length $\times$ width $\times$ thickness). The optical (transparent cover transmittance $\tau=$ $0.9 \%$, absorptance of the glass covers the visible range $\alpha_{\mathrm{g}}=$ $0.05)$ and thermal properties $(\lambda=0.19 \mathrm{~W} / \mathrm{m} . \mathrm{K})$ of the Plexiglas plate reduced convective losses from the absorber plate and adsorption tube by retaining the stagnant air layer between the absorber plate and the cover glass. The plate also creates a greenhouse effect by allowing short-wave radiation to be absorbed from the sun and nearly opaque to long-wave thermal radiation to be emitted by the absorber plate to the atmosphere. A blackened (solar absorptance $\alpha=0.96$, infrared emittance $\mathrm{P}=0.04$, reflectance of coated surfaces $\varepsilon=0.88$ ) absorber plate (aluminium, $0.5 \mathrm{~mm}$ thick, $\lambda=210 \mathrm{~W} / \mathrm{m} . \mathrm{K}$ ) with attached absorber tubes (copper, $\lambda=380$ ) is used to maximize radiant energy absorption, transfer this heat to a working fluid at a minimum temperature difference, and minimize radiant emission.

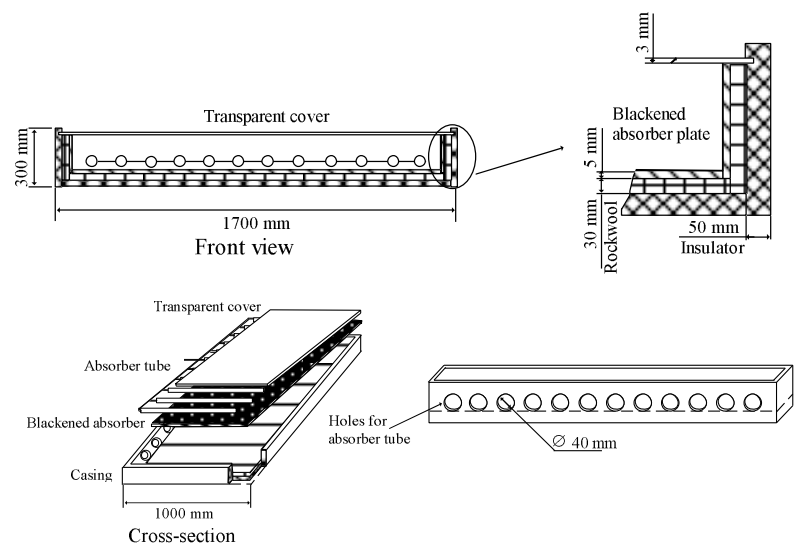

Fig 1: Components of the solar collector

\subsection{Solar Collector Adsorber Tube}

A adsorber copper tube (Ø $15 \mathrm{~mm})$ is placed inside a vacuumsealed absorber tube (Ø $40 \mathrm{~mm})$ and attached to a blackened absorber plate (Fig. 2a). The space between the adsorber tube and absorber tube is filled with activated carbon fiber (AK type; Unitika Ltd., Japan) and methanol. The lengths of the adsorber tube and absorber tube are $1010 \mathrm{~mm}$ and $1000 \mathrm{~mm}$, respectively. The adsorber tube is perforated with $0.3 \mathrm{~mm}$ holes, is hollow, and the space inside is evacuated. The reason for a perforated adsorber tube is to promote a change in state of the liquid methanol inside the absorber tube and to ease methanol flow to and from the activated carbon fiber. When solar radiation falls on the surface of the absorber plate and absorber tube, the liquid within the adsorption tube heatsed, turns to hot vapor, and is later evacuated. The casing contains twelve holes into which the adsorption tubes with adsorber tubes are fitted and the closed ends are placed inside the casing (Fig. 2b).

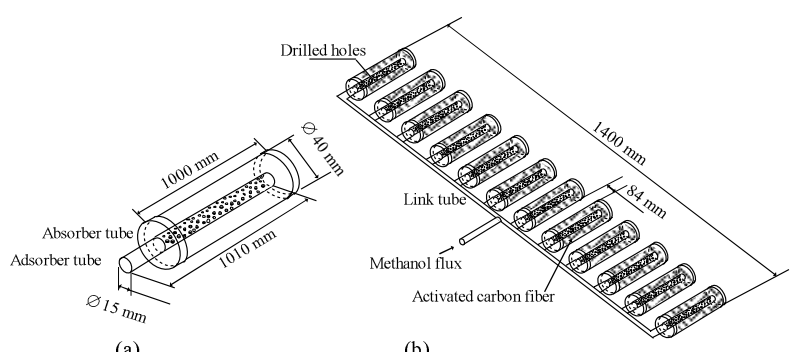

Fig 2: Schematic diagram of cross-section of one ended absorber: adsorber tube (a) and link tubes along the length (b)

\subsection{Physical Processes inside a Flat-Plate Solar Collector}

Figure 3 shows a schematic drawing of the heat flow through a solar collector [9].

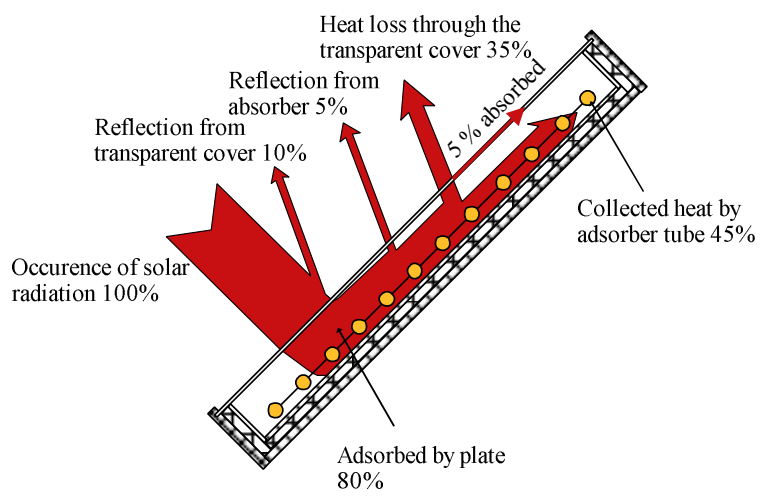

Fig 3: Heat flow through a flat-plate solar collector

\subsection{The Solar Collector Energy Balance}

The mathematical model of the flat-plate solar collector is based on the energy balance of the collector absorber plate [10]. The total solar radiation $\dot{G}_{s}$ incident on the flat plate solar collector has three components: direct beam solar radiation $\dot{G}_{b}$, ground diffuse radiation $\dot{G}_{g}$, and sky reflection radiation $\dot{G}_{d}$. However, this amount of radiation is reduced by optical losses from the flat-plate solar collector. The actual amount of radiation from the sun that is available for the collector $\dot{G}$ is given by

$\dot{G}_{s}=\dot{G}_{b}(\tau \cdot \alpha)_{b}+\dot{G}_{g}(\tau \cdot \alpha)_{g}+\dot{G}_{d}(\tau \cdot \alpha)_{d}$

$(\tau \cdot \alpha)_{b},(\tau \cdot \alpha)_{g},(\tau \cdot \alpha)_{d}$ are the transmittance-absorptance product for beam, ground, and sky diffuse radiation, respectively. The useful amount of radiation $\dot{Q}_{n e t}$ that goes to the adsorption bed is reduced by heat losses to the ambient air $\dot{Q}_{i}$. For collector plate area A, useful heat $\dot{Q}_{n e t}$ is calculated from the following equation:

$\dot{Q}_{n e t}=A \cdot \dot{G}_{s}-\dot{Q}_{i}$

Heat loss from the solar collector is expressed in terms of the overall heat transfer loss coefficient $\mathrm{U}$ as follows: $\dot{Q}_{i}=U$. $A\left(T-T_{a m b}\right)$ 
Heat transfer from the lateral and bottom surfaces of the solar collector are negligible compared with heat losses from the top surface of the collector. The heat transfer coefficient is calculated from the method presented by Klein [11] and is discussed by Duffie and Beckman [12] as follows:

$U=\left[\lambda+\frac{1}{U_{w}}\right]^{-1}+\frac{\sigma}{\xi}\left(T+T_{a m b}\right) \cdot\left(T^{2}+T_{a m b}^{2}\right)$

where,

$$
\begin{aligned}
& \lambda=\frac{N \cdot T}{\Gamma}\left[\frac{\left(T+T_{a m b}\right)}{N+\varphi}\right]^{-\omega} \\
& \omega=0.43\left[1-\frac{100}{T}\right] \\
& \xi=\frac{1}{\varepsilon_{p l}+0.0591 N \cdot U_{w}}+\frac{2 N+\varphi-1+0.133 \varepsilon_{p l}}{\varepsilon_{g c}}-N \\
& \varphi=\left(1+0.089 U_{w} \cdot \varepsilon_{p l}\right) \cdot(1+0.07866 N)
\end{aligned}
$$

The wind convection coefficient $U_{w}$ is in terms of the wind velocity $V_{w}$ from the following equation [13]:

$U_{w}=5.7+3.8 V_{w}$

When the flat-plate solar collector glass cover is opened during the isosteric cooling and adsorption processes, the overall heat transfer coefficient is given by

$U=\varepsilon_{p l} \cdot \sigma\left(T+T_{a m b}\right) \cdot\left(T^{2}+T_{a m b}^{2}\right)+U_{w}$

A: Area, $\mathrm{m}^{2}$

$\mathrm{T}_{\mathrm{amb}}$ : Ambient temperature, ${ }^{\circ} \mathrm{C}$

$\mathrm{N}$ : Number of transparent covers

$\sigma$ : Stefan-Boltzmann constant

$\varepsilon_{p l}$ : Absorber plate emissivity

$\varepsilon_{g c}$ : Transparent cover emissivity

$U:$ Overall heat transfer coefficient

$\lambda$ : Wavelength

$\tau$ : Transmittance

$\omega$ : Hour angle, ${ }^{0}$

$\varphi$ : Latitude (angle defined locally), ${ }^{0}$

\subsection{Measurements}

The heating performance test of the experimental solar collector was undertaken during the period from July 1 to December 31, 2014 to determine the influence of the uncontrolled natural factor (solar radiation) and manipulating factor (adsorber tube temperature). Solar radiation and its diurnal changes were investigated to identify the dynamic response of the adsorber tube temperature. Temperature was measured using two 47SD digital thermometers with data logger function (Sato Shoji Inc., Japan), each with four thermocouples (Ø $0.3 \mathrm{~mm}$ ) having an accuracy of $\pm 0.1{ }^{\circ} \mathrm{C}$. Solar radiation was measured using a solar power meter with data logger function (SPM-SD; Sato Shoji Inc., Japan). These data were recorded at intervals of $60 \mathrm{~s}$ for $24 \mathrm{~h}$ resulting in 1440 readings per day. A NARX model, with Bayesian training algorithms, was examined and compared using MATLAB ${ }^{\circledR}$ environment (version R2014a, The MathWorks Inc., Natick, MA, USA) and the Neural Network Toolbox ${ }^{\mathrm{TM}}$.

\subsection{Modelling the Solar Collectors with NARX}

In dynamic networks, the output depends both on the current input to the network and on the current or previous inputs, outputs, or states of the network. Generally, time series is a sequence of discrete data taken at specific time intervals, for example, daily time series, or monthly, etc. However, time series depend heavily on past values. A normal feed-forward network with a small number of inputs compared with the length of the time series can estimate the next value counting on little factors. In this paper, the architectural approach proposed to deal with a single input-single output chaotic time series is based on a recurrent dynamic network, with feedback connections enclosing several layers of the network called "Nonlinear AutoRegressive models with eXogenous input (NARX model)". A NARX neural net has been demonstrated to be well suited for modeling nonlinear systems and especially time series $[8,14]$. A Nonlinear AutoRegressive recurrent neural network with eXogenous inputs model is given as follows [15]:

$y(t)=f(x(t), \cdots, x(t-a), y(t-1), \cdots, y(t-b), d(t-$ 1), $\cdots, d(t-b))$

where $\mathrm{d}$ is the target (predict) for the time series; $\mathrm{y}$ is the past predicted value; $a, b$ are the input and output order; $x$ is the exogenous variables; and $\mathrm{f}$ is a nonlinear function.

In the model [16], the input order gives the number of past exogenous variables that are fed into the system. The values for the exogenous variables are from current time $t$ to $t-a$, where the input order is a. The input variables with their order are called the input regressor. The past predicted value is $y$ and to predict the value at current time $t$, values starting from $\mathrm{t}-1$ to $\mathrm{t}-\mathrm{b}$ are used, where the number of past predictions fed into the model is $b$ (the output order) and called the output regressor. The real value of the time series $d$ (predicted target) is also fed into the system. The same order as for past predicted values is also used. If these values are missing, the system tries to predict the next values of the time series from the exogenous variables only or uses the feedback from past predicted values [16]. $\mathrm{M}$ is the number of inputs (exogenous). If $M$ is 0 , the NARX can fall back to a prediction system without exogenous inputs. The input order based on how many previous values are given to the system is denoted by a. Another parameter $b$ is the actual order of delayed outputs or delayed targets. The parameter $\mathrm{i}$ represents a certain exogenous variable taken into account [16] and $\mathrm{x}(\mathrm{t})$ actually represents a vector of exogenous variables at time $t$, with $i$ varying from 1 to $M$. The vector of outputs denoted by $y$ and $\mathrm{N}$ is the number of output variables. The system parameters are given as follows:

Exogenous inputs : M;

Predicted Outputs : N;

Input delays order: $\mathrm{a}>0$ only if $\mathrm{M}>0$;

Output delay order: $\mathrm{b}$

The prediction, is $y(t)$ when the output of the network for time is $\mathrm{t}$, and the target $\mathrm{d}(\mathrm{t})$ can compute the error $\mathrm{e}(\mathrm{t})$ as $\mathrm{d}(\mathrm{t})-\mathrm{y}(\mathrm{t})$. Figure 6 shows a standard closed feedback loop NARX network architecture. 

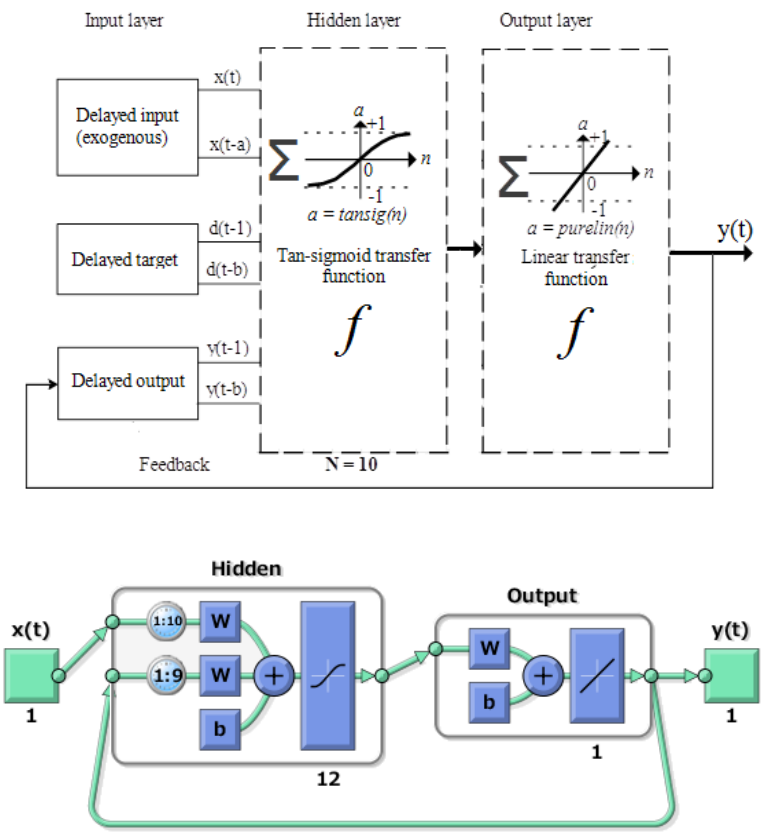

Fig 4: A single input-output closed feedback loop NARX network architecture

\subsection{Number of Hidden Layers and Neurons}

Cybenko [17] showed that any multi-dimensional nonlinear mapping of any continuous function can be carried out by a two-layer model with a suitable chosen number of neurons in its hidden layer. The number of neurons indicates the complexity that can be approximated by the neural network. It is desirable to use the simplest possible network structure with the least number of input parameters. The developed model can be utilized to validate new process measurements. A true neural network training procedure is usually based on an iterative approximation in which the parameters are successively updated in numerous steps. Such steps can be based on a single data item, on a set of them, or on all available data points. In each step, the desired outcome is compared with the actual outcome and, using the knowledge of the architecture, all parameters are changed slightly such that the error for the presented data points decreases [18]. Although increasing the number of neurons is sometimes necessary to catch nonlinear dynamics of the system, it does not mean that it can always and necessarily improve model accuracy and generalizability [19].

\subsection{Weight Values}

Before training an ANN, the initial values of weights and biases have to be determined automatically by the ANN Toolbox software or it can be adjusted manually through writing and running codes in MATLAB ${ }^{\circledR}$ environment.

\subsection{Training Algorithms}

The network training function that updates the weight and bias values according to Levenberg-Marquardt optimization was modified to include the regularization technique. It minimizes a combination of squared errors and weights and then determines the correct combination to produce a network that generalizes well. The process is called Bayesian regularization [16]. If the number of weights in the network increases, the advantage of this algorithm decreases. However, in Bayesian regularization, training stops according to adaptive weight minimization (regularization). According to Beale et al. [18], the advantages of Bayesian regularization are as follows:

- The network training function updates weight and bias values according to gradient descent with momentum

-The network training function updates weight and bias values according to gradient descent with adaptive learning rate

-The network training function updates weight and bias values according to gradient descent momentum and adaptive learning rate.

The neural network training can be made more efficient if certain preprocessing steps on the network inputs and targets are performed. The normalization of the input and target values into the interval $[-1,1]$. This simplifies the problem of the outliers for the network. The normalized inputs and targets returned all fall in the interval $[-1,1]$.

\subsection{Transfer Functions}

Karlik and Olgac [20] stated that transfer (activation) functions transform neuron into an output signal. TanSigmoid and Linear transfer functions are the two common transfer functions that are employed for a multi-layer model. These functions are differentiable and can cope with nonlinearity of the complex systems.

\subsection{Network Identification through Training - Testing - Validation}

The procedures for NARX model identification is shown in Fig. 7. This model identification process includes the following [21]:

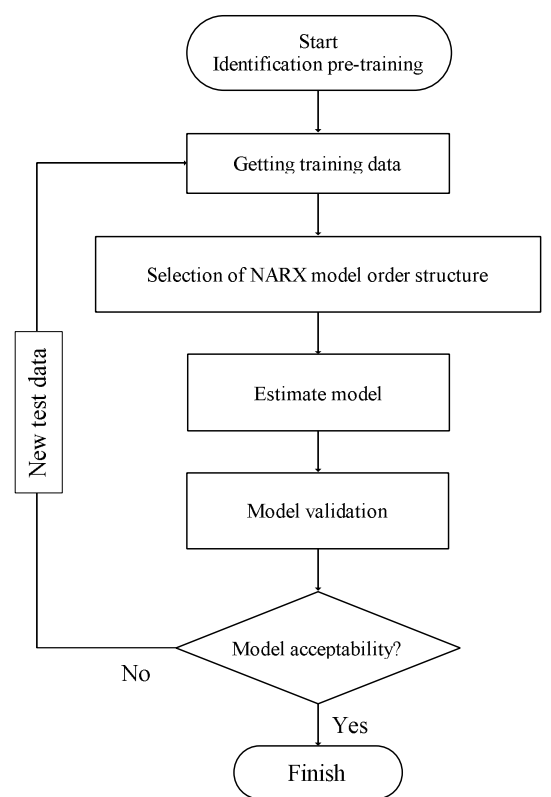

Fig 5: Flow chart for training of NARX neural network model

\subsubsection{Identification pre-training}

This study is very important in choosing the controlled, manipulated, and disturbance variables.

\subsubsection{Getting training data}

Studying the input range is required to calculate the maximal possible values of all input signals so that both inputs and outputs will be within the desired operating conditions range. The selection of input signal would allow the incorporation of additional objectives and constraints, that is, minimum or 
maximum input event separations which are desirable for the input signals and the resulting process behavior. In this experiment, a previously obtained experimental data set, which consisted of 290 input vectors and their corresponding output vectors, was divided randomly into three subsets. Among this data, the first subset is the training set (75\%), which is used for computing the gradient and updating the network weights and biases to minimize the network performance function. Training in the neural network is the process by which a neural network learns to recognize the underlying relationship between inputs and output, or just among the inputs. The second subset is a validation set (15\%) and validation error is monitored during the training process. The validation error decreases during the initial phase of training, as does the training set error. The network weights and biases are saved at the minimum validation set error. And finally, the test set (15\%) is used after training and validation for a final test. In most instances, such testing techniques prove adequate for the acceptance of a neural network system. The validation data set is used to stop training early if further training on the primary data will hurt generalization of the validation data. Test vector performance can be used to measure how well the network generalizes beyond primary and validation data. When the training is complete, network performance can be checked to see if any changes need to be made to the training process, the network architecture, or the data sets [18].

\subsubsection{Estimate model}

The important step in estimating NARX models is to choose the model order. A tangent sigmoid function was applied to the hidden layer, and a linear transfer function was used in the output layer. The whole process was repeated for over 1000 iterations. The neuron number of the hidden layer for estimating the model was selected as 12 (two tap delay lines: $\mathrm{d} 1=10, \mathrm{~d} 2=9$ ) with the following trial and error technique. The model performance was evaluated by mean squared error (RMSE) and coefficient of determination ( ${ }^{2}$ ) [18].

$$
\begin{aligned}
& M S E=\frac{1}{n} \cdot \sum_{i=1}^{n}\left(y_{k}-r_{k}\right)^{2} \\
& R^{2}=1-\left(\frac{\sum_{i=1}^{n}\left(y_{k}-r_{k}\right)^{2}}{\sum_{i=1}^{n}\left(y_{k}\right)^{2}}\right)
\end{aligned}
$$

where $n$ is the number of data points, $\mathrm{y}_{\mathrm{k}}$ is network output, and $r_{k}$ is desired target. The optimum ANN configuration that gave the lowest MSE (near 0 means a close relationship, 1 a random relationship) and highest $\mathrm{R}^{2}$ (an $\mathrm{R}$ value of 1 means a close relationship, 0) a random relationship) value for the training dataset was selected.

\subsubsection{Model validation}

When training a neural network, a stop criterion is determined to avoid overfitting, because an ANN has the potential tendency to overfit during training. Overfitting can occur during training when the ANN gets too specialized to fit the training data extremely well, but at the expense of reasonably fitting the validation data. Overfitting is reflected by a steady increase in validation error accompanied by a concomitant steady decrease in training error. Poor performance due to overfitting is one of the most common problems in training ANNs, but it can be overcome by using the cross-validation method, decreasing the number of neurons in hidden layer(s), or adding a penalty term to the objective function for large weights. By using the cross-validation method, network performance is measured during training and if any incentive is given, the training is stopped before the maximum number of epochs is reached. Epoch is a neural network term for iteration in a training process. The number of epochs shows the number of times that all patterns are presented to the neural network. More epochs means more training time. In each epoch of an ANN, all the weight values of the neurons are updated [19]. The model was validated with validation data that were independent data sets not used in NARX model parameter estimation.

\subsubsection{Model acceptance}

The objective in training a neural network is to minimize errors as much as possible. Minimizing errors simply means improving the performance of the training and getting a more accurate model.

\section{RESULTS AND DISCUSSION 3.1 Fundamental Responses of inside Temperature to Manipulating Factors}

Figure 6 shows the short-term dynamic response of solar collector adsorber tube temperature to solar radiation, which fluctuated because of the weather conditions (sunny-cloudy). After sunrise, when solar radiation started increasing, the adsorber tube temperature also started increasing. The temperature inside the adsorber tube steadily increased because of the high thermal conductivity of the copper adsorber tube and high thermal absorbance of the activated carbon fiber stored inside the adsorber tube. When the solar radiation started decreasing, the adsorber tube temperature also decreased. This result shows that solar radiation caused the temperature difference in the absorber tube. Therefore, we chose solar radiation as the input variable for system identification. Place Tables/Figures/Images in text as close to the reference as possible (see Figure 1). It may extend across both columns to a maximum width of $17.78 \mathrm{~cm}$ (7').

\subsection{Solar Collector Adsorber Tube Temperature for Identification}

Many types of data-sets for the input and output variables were obtained for identification from a real system. Figure 7 shows typical diurnal changes in solar radiation and the solar collector adsorber tube temperature of the solar collector. From the figure, it is observed that solar radiation first increased the solar collector adsorber tube temperature, and then in the absence of solar radiation the solar collector adsorber tube temperature decreased. Twelve types of data sets of the inputs and the output were obtained for identification. During the experiment period, under a maximum solar radiation of $669 \mathrm{~W} \cdot \mathrm{m}^{-2}$, the adsorber tube temperature was $86^{\circ} \mathrm{C}$, while after sunset the average temperature was $18^{\circ} \mathrm{C}$. 

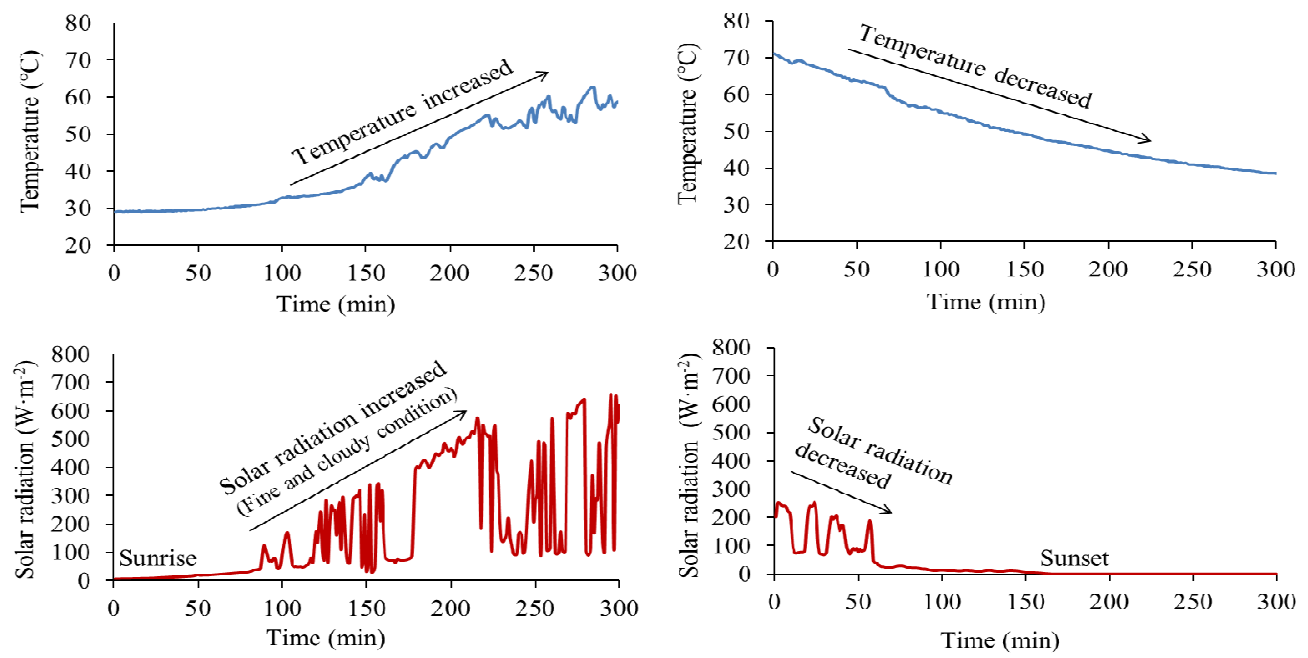

Fig 6: Relationship between solar radiation and solar collector adsorber tube temperature
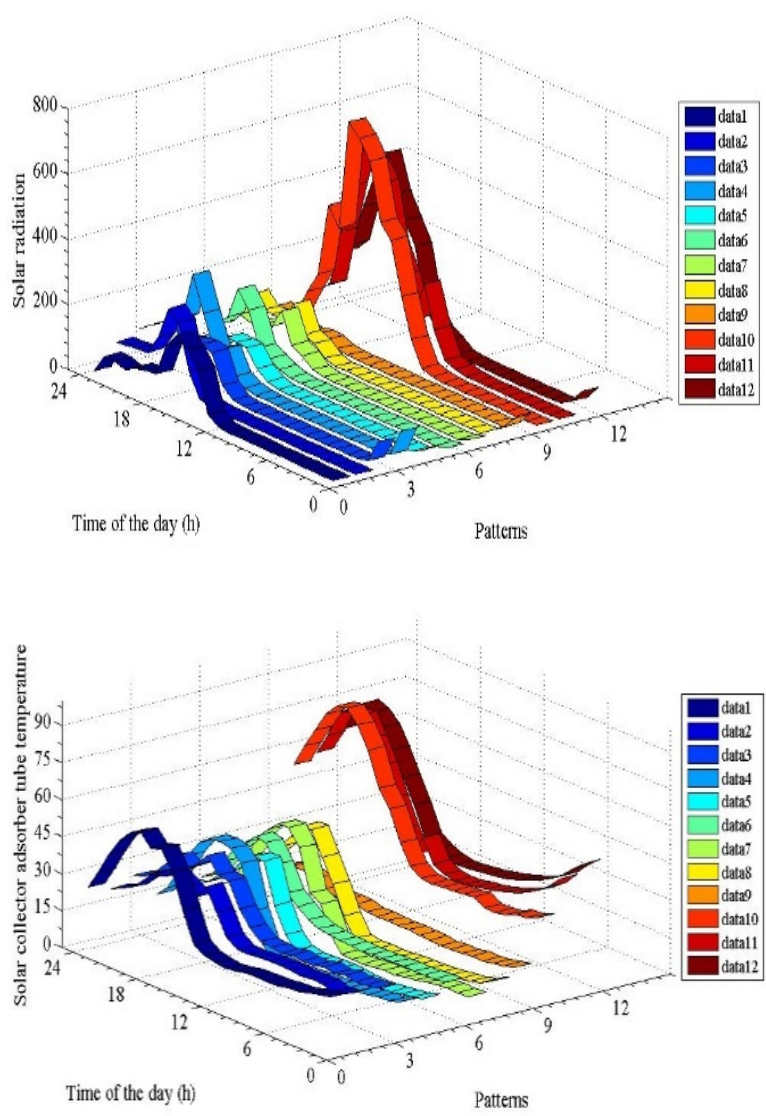

Fig 7: Typical diurnal changes in solar collector adsorber tube temperature, as affected by solar radiation (12 patterns)

\subsection{Training Performance Analysis}

To find the best model for the solar collector, the generated code was run in MATLAB and a plot of the best resulting network based on the average performance of training and test errors with the number of training epochs is shown in Fig. 8. The graph shows that the large values for the MSE of the network decrease to a smaller value as the weights are improved, that is, network training. Training stopped according to adaptive weight minimization at 35 epochs, that is, Bayesian regularization [18].

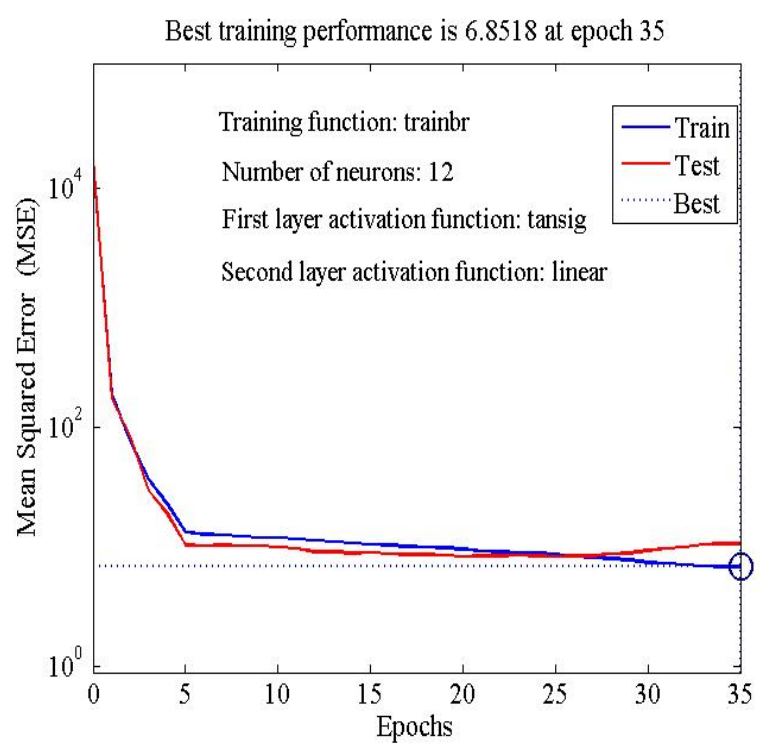

Fig 8: Performance of the ANN model

Figure 9 shows the regression plot, in which the circles are the data points and the line represents the best fit between outputs and targets. As Fig. 9 shows, R values for all graphs are very close to 1 . Therefore, it can be concluded that the ANN structure is satisfactory in predicting the solar collector adsorber tube temperature by solar radiation.

\subsection{Prediction Performance Analysis}

Figure 10a, b, c show the predicting performance of the NARX model by evaluating time series response, error autocorrelation, and input error cross-correlation parameters. Time series response displays the inputs, targets, and errors versus time, and can also indicate which time points were selected for training, testing, and validation. The error autocorrelation and input error cross-correlation illustrated in Fig. 10b, c show how the errors correlate with the input sequence; for a perfect prediction model, all the correlations should fall within the confidence bounds around zero. This observation suggests that the NARX ANN trained with the training algorithm 'Bayesian' is efficient in predicting the solar collector adsorber tube temperature with solar radiation. 

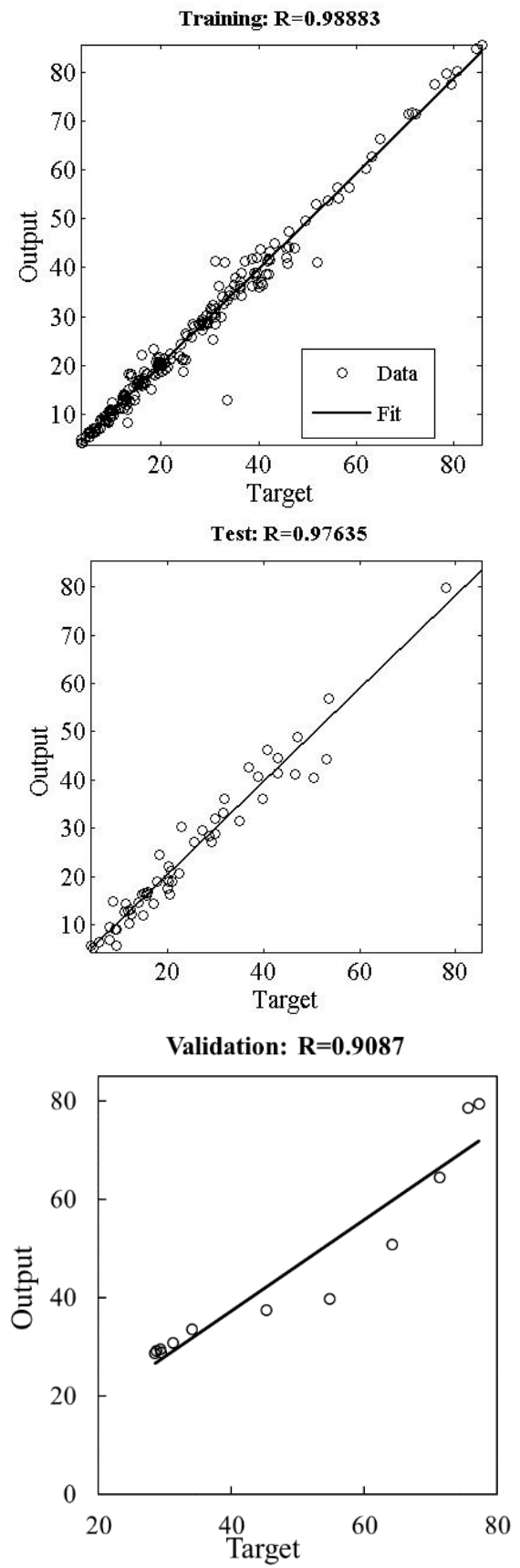

Fig 9: Regression analysis with training test and validation for the optimum ANN model

Figure 11 shows the comparison of the estimated and observed relationship between solar radiation and the solar collector adsorber tube temperature. The estimated relationship was obtained from a simulation of the $\mathrm{NN}$ model. All the data in this graph were obtained from the calculation of the step responses of the adsorber tube temperature as affected by various levels of the step input of the solar radiation. It can be seen that the estimated relationship was closely related to the observed one. In general, it is well known that the relationship between solar radiation and the net solar collector adsorber tube temperature shows nonlinear characteristics because of the thermo-physical properties of the adsorber tube and environmental. The nonlinear characteristics appear in both curves. This implies that the nonlinear identification of the net solar collector adsorber tube temperature was well attained using the $\mathrm{NN}$ method.

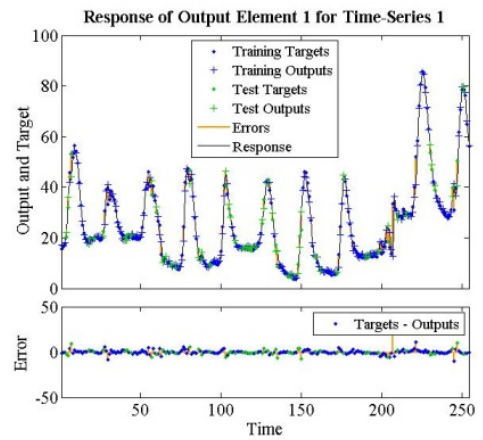

(a)

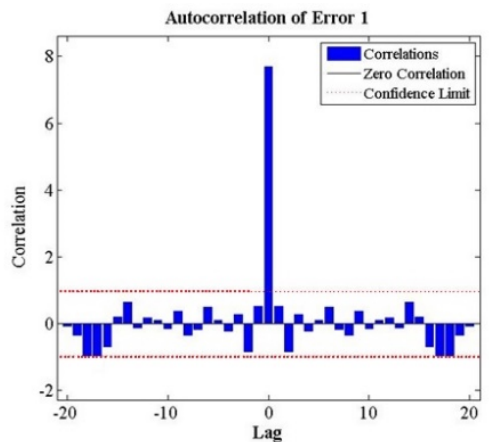

(b)

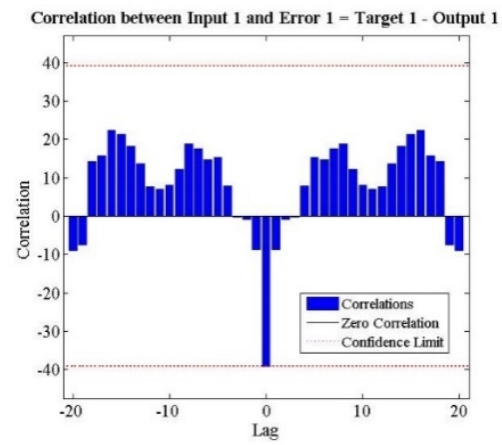

(c)

Fig 10: Prediction performance analysis of NARX model

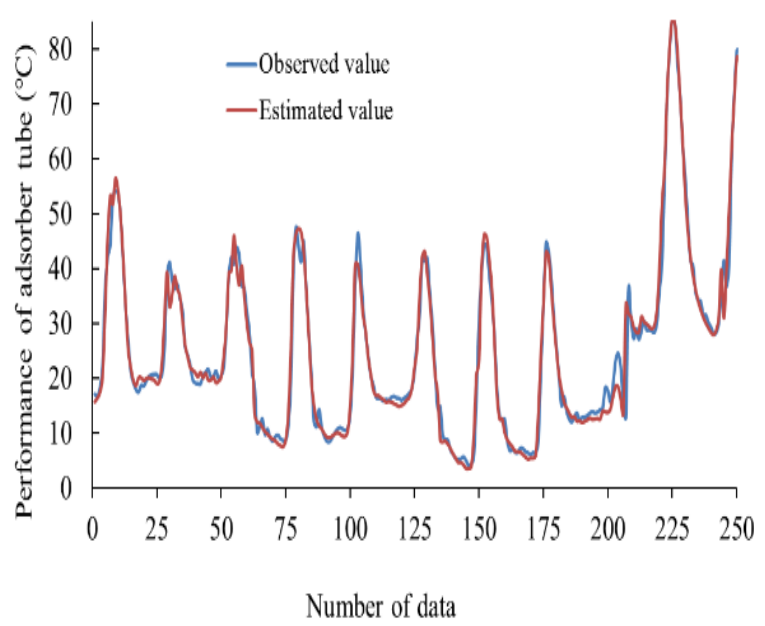

Fig 11: Simulated dynamic nonlinear relationship between observed and estimated results 
The observed and estimated relationships between solar radiation and solar collector adsorber tube temperature are shown in Fig. 12. These values are obtained from the stationery values of step responses of net solar collector adsorber tube temperature as affected by the various gain of step input of solar radiation by using the nonlinear dynamic model method. It can be seen that the estimated relationship was closely related to the observed one. These results suggest that a reliable computational model could be obtained for predicting the behavior of the adsorber tube temperature under any combination of solar radiation.
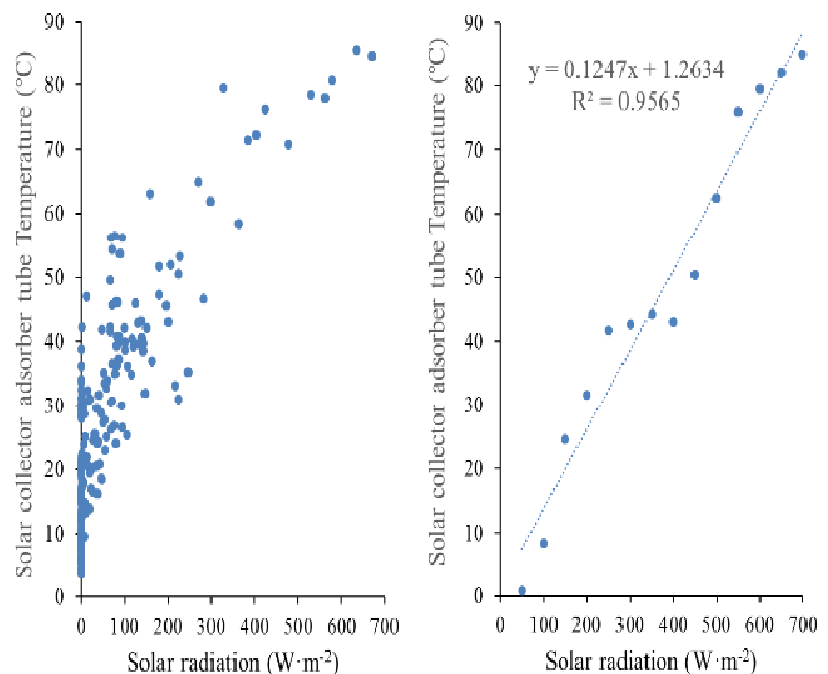

Fig 12: Predicted satic nonlinear relationships between observed and estimated results

\section{CONCLUSION}

The advantages of the NARX-based ANN are speed, simplicity, and capacity to train from example over the mathematical models. The preprocessing data have been used to develop an ANN model to characterize the thermal behavior of the solar collector adsorber tube. In this paper, the performance of the prediction for different time series was tested using a NARX Bayesian regulation. The comparative analysis between the measured data and estimated data showed that the NARX model can recognize the relationship between the input and output variables and can accurately predict hourly solar collector adsorber tube temperature with NARX 1-12-1 (input-hidden neuron number-output) with tapped delay lines $(\mathrm{d} 1=10, \mathrm{~d} 2=9)$. Therefore, in this study we can conclude that the Bayesian training algorithm with a minimum mean squared error (MSE near zero) and maximum correlation coefficient ( $\mathrm{R}$ near 1 ) was found to be the best under 35 epochs in the training, testing, and validation period for predicting the solar collector adsorber tube thermal performance. Therefore, NARX-based ANN can successfully be used for the prediction of thermal performance of a solar adsorber tube by reducing time in testing of prototypes for longer duration.

\section{ACKNOWLEDGMENTS}

We would like to acknowledge the financial support provided by the Grants-in-Aid for Scientific Research from the Japan Society for the Promotion of Science (No. 26450354) and Grant for Basic Science Research Projects from the Sumitomo Foundation (No. 143039).

\section{REFERENCES}

[1] Balzani M. and Reatti A. 2005. Neural network bases model of a PV array for the optimum performance of PV system. Proceeding of IEEE international Conference on Microelectronics and electronics, 123-126.

[2] Kalogirou S.A. 2004a. Solar thermal collectors and applications. Progress in Energy and Combustion Science, 30(3): 231-295.

[3] McCulloch W.S. and Pitts W. 1943. A logical calculus of the ideas immanent in nervous activity. Bulletin of Mathematical Biophysics, 5: 115-133.

[4] Kalogirou S.A. 2005. Artificial intelligence in renewable energy application in buildings. Proceedings of the international conference on the integration of the renewable energy systems (RES) into the building structures, Patra, Greece, 112-126.

[5] Toriman M.E., Juahir H., Mokhtar M., Barzani G.M. and Mastura S.A.S. 2009. Predicting for discharge characteristics in Langat River, Malaysia using neural network application model. Research Journal of Earth Science, 1: 15-21.

[6] Yang K.T. 2008. Artificial neural networks (ANNs): a new paradigm for thermal science and engineering. Journal of Heat Transfer, 130(9): 19pp. DOI: $10.1115 / 1.2944238$

[7] Lin T., Horne B.G., Tino P. and Giles C.L. 1996 Learning long-term dependencies in NARX recurrent neural networks. IEEE Transactions on Neural Networks, 7(6): 1329-1351

[8] Diaconescu E. 2008. The use of NARX neural networks to predict chaotic time series. WSEAS transactions on computer research, Volume 3(3): 182-191.

[9] Struckmann F. 2008. Analysis of a flat-plate solar collector. Project Report, MVK160 Heat and Mass Transport, Lund, Sweden.

[10] Hassan H.Z. 2013. A solar powered adsorption freezer: A Case Study for Egypt's Climate. International Journal of Energy Engineering, 3(1): 21-29. DOI: 10.5923/j.ijee.20130301.04

[11] Klein S.A. 1975. Calculation of flat-plate collector loss coefficients. Solar Energy, 17: 79-80.

[12] Duffie J.A. and Beckman W.A. 2006. Solar engineering of thermal processes. 3 ed. New York: John Wiley \& Sons.

[13] Saraf G.R. and Hamad F.A.W. 1988. Optimum tilt angle for a flat plate solar collector. Energy Conversion and Management, 28(2): 185-191.

[14] Gao Y. and Joo E.M. 2005. NARMAX time series model prediction: feedforward and recurrent fuzzy neural network approaches. Fuzzy Sets and Systems, 150(2): 331-350.

[15] Siegelmann H.T., Horne B.G. and Giles C.L. 1997. Computational capabilities of recurrent NARX neural networks. IEEE transactions on systems, man, and cybernetics. Part B, Cybernetics: a publication of the IEEE Systems, Man, and Cybernetics Society, 27(2): 208-215. 
[16] Eugen H. 2012. NARX neural networks for sequence processing tasks. Master of Science Thesis Universitat Politècnica de Catalunya - Universitat Rovira i Virgili Universitat de Barcelona.

[17] Cybenko G. 1989, Approximation by superpositions of a sigmoidal function. Mathematics of Control, Signals, and Systems, 2(4): 303-314.

[18] Beale M.H., Hagan M.T. and Demuth H.B. 2014. Neural Network Toolbox ${ }^{\mathrm{TM}}$ User's Guide, R2014a ed., Natick, MA: The MathWorks.

[19] Asgari H. 2014. Modelling, Simulation and control of gas turbines using artificial neural networks. PhD thesis
Mechanical Engineering University of Canterbury Christchurch, New Zealand.

[20] Karlik B. and Olgac A.V. 2010. Performance analysis of various activation functions in generalized MLP architectures of neural networks. International Journal of Artificial Intelligence and Expert Systems (IJAE), 1(4): 111-122.

[21] Zulkeflee S.A., Suhairi A.S. and Norashid A. 2011. Nonlinear autoregressive with Exogenous inputs based model predictive control for batch citronellyl laurate esterification reactor, Advanced Model Predictive Control, Dr. Tao ZHENG (Ed.), ISBN: 978-953-307298-2, InTech. DOI: 10.5772/16963. 